\title{
Análisis de percepción sobre la nanociencia y la nanotecnología: el caso de la comunidad universitaria de UAM, UDLAP E IPN'
}

\author{
José Manuel Mata Méndez y Judith Sarai Peña Jiménez*
}

\begin{abstract}
Resumen: Se presenta el análisis de percepción social de las comunidades universitarias de los planteles Universidad Autónoma Metropolitana Iztapalapa, Universidad de las Américas Puebla, Escuela Superior de Ingeniería Mecánica y Eléctrica Culhuacán, Escuela Superior de Comercio y Administración Tepepan y Centro de Investigación y de Estudios Avanzados sedes Norte y Sur, sobre el estado e implicaciones de la nanociencia y la nanotecnología a partir de los datos obtenidos tras una encuesta que se realizó a 464 estudiantes y profesores de las 7 sedes mencionadas. Los datos siguen la estructura de la encuesta realizada en el volumen 4, número 1 , de esta revista, siendo modificados sólo algunos datos que podrán verse en el anexo de tal suerte que la encuesta fuera generalizable a todas las instituciones encuestadas. Concluimos reiterando el interés por el fortalecimiento al estímulo en la ciencia y la tecnología en nuestro país con responsabilidad social. Estudios como éste pretenden detectar el (des)interés en la ciencia y la tecnología de la comunidad universitaria y los modos en que es percibida. El objeto: contribuir con un análisis útil para cualquier intento que pretenda revertir la desinformación.

Palabras clave: nanociencia, nanotecnología, percepción social, implicaciones, regulación.
\end{abstract}

ABSTRACT: Analysis of social perception on nanoscience and nanotechnology state of the art and implications is presented as a result of a wide survey carried out at Universidad Autónoma Metropolitana Iztapalapa, Universidad de las Américas Puebla, Escuela Superior de Ingeniería Mecánica y Eléctrica Culhuacán, Escuela Superior de Comercio y Administración Tepepan and the Centro de Investigación y de Estudios Avanzados, north and south units. Four hundred and 64 interviews were applied to students and teachers.

Data follows the survey scheme used on a previous study for UNAM's Ciudad Universitaria campus social perception that has been published on volume 4, No. 1 of this journal. Some small modifications have been done in order to fit the survey to all institutes and universities contexts. We conclude by supporting the effortand interest of promoting responsible science and technology in our country. Studies like this have the purpose of detecting the degree of (dis)interest on science and technology of the high education level community and the ways its development is perceived. The goal: Contributing with an analysis considered useful for any attempt to revert disinformation. Key words: nanoscience, nanotechnology, social perception, implications, regulation.

\section{INTRODUCCIÓN}

En enero de 2012 se revisó el contenido de la encuesta realizada a la comunidad de Ciudad Universitaria de la Universidad Nacional Autónoma de México (UNAM) para conocer su percepción sobre la nanociencia y la nanotecnología (véase: Delgado y

\footnotetext{
1 El trabajo presenta los resultados de trabajo de campo sobre percepción social realizado en el marcodel proyecto "LABnano - Laboratorio SocioEconómico en Nanociencia y Nanotecnología" (CEIICH-CONACYT, apoyo complementario 2009, No. 118244)

* Tesistas de la licenciatura en sociología de la Facultad de Ciencias Políticas ySociales de la UNAM. Colaboradores del proyecto LABnano<www.labnano.ceiich.unam.mx>.
} 
Peña, 2011). Aunque los resultados coincidían con las tendencias internacionales, se resolvió pertinente la aplicación de dicho estudio, con el objeto de incluir a otras instituciones universitarias y realizar así un análisis comparativo.

La nueva fase de estudio de percepción social y que a continuación se presenta incluyó la aplicación de 464 encuestas al estudiantado y docentes de los planteles Universidad Autónoma Metropolitana Iztapalapa, Universidad de las Américas Puebla, Escuela Superior de Ingeniería Mecánica y Eléctrica Culhuacán, Escuela Superior de Comercio y Administración Tepepan y Centro de Investigación y de Estudios Avanzados sedes Norte y Sur. La composición por género fue de 55\% mujeres y 46\% hombres.

Los aspectos generales sobre la percepción de la comunidad universitaria de las distintas instituciones tienden a ser relativamente similares a los obtenidos en la encuesta realizada en 2011 en Ciudad Universitaria de la UNAM. La principal diferencia radica en que en las nuevas sedes se presentó una alta tasa de desinterés respecto a las preguntas abiertas, salvo en los casos de CINVESTAV Norte y la UDLAP. Del mismo modo se presentó una gran indiferencia al momento de llenar el denominado cuadro de confianza respecto a productos nano en tanto que en muchas ocasiones resultó en blanco.

Pese a tal situación, se pudo constatar que la idea generalizada es que la nanotecnología es una tecnología a pequeña escala, que no existen las condiciones necesarias para que México innove en este rubro debido a la falta de apoyo por parte del gobierno, a pesar de que sí existe el potencial humano necesario para llevarlo a cabo. Igualmente, se considera necesario el etiquetado de los productos nano y que la regulación de importaciones es importante.

En el caso de la encuesta de la UNAM, previamente a la aplicación de las encuestas se proporcionó un folleto y una hoja con definiciones de la nanociencia y la nanotecnología a modo de introducción a la encuesta. En esta ocasión se decidió que el proceso fuera diferente, ya que la metodología y tratamiento analítico también lo fueron en tanto que se trató fundamentalmente con estudiantes pertenecientes a instituciones cuya especialidad es el área de ciencias exactas (excluyendo el caso de la UAM). En tal sentido se consideró conveniente no presentar material algunode modo tal que se pudiera apreciar el conocimiento real que los actores de ciencia poseen en cada institución. Pese a ello, antes de iniciar la encuesta se les ofreció una breve explicación y de igual forma la posibilidad de preguntarnos directamente en caso de persistir alguna duda. Como se observa en las gráficas no hubo mayor interés en la temática o los aspectos de la encuesta más que en dos ocasiones.

\section{ANÁLISIS DE RESULTADOS POR INSTITUCIÓN UAM-I}

La encuesta escrita fue aplicada entre los meses de marzo y abril a estudiantes de la Universidad Autónoma Metropolitana, plantel Iztapalapa (UAM-I). Al igual que en el estudio realizado en la UNAM, se dividieron a los estudiantes de la UAM-I en dos grupos: el primero lo conforman 105 alumnos de carreras pertenecientes al área de las ciencias naturales y exactas, mientras que el segundo grupo lo forman 81 alumnos del área de las ciencias sociales y humanidades, para un total de 186 alumnos encuestados. La gran mayoría se encuentra entre los 18 y los 24 años de edad. 
De los estudiantes de las carreras de ciencias naturales y exactas, la que más concentración tiene es la licenciatura en biología con un 39\% respecto al total, seguido por la licenciatura en matemáticas con un 14\% y la licenciatura en computación con un $13 \%$, completando la gráfica aparecen otras ocho carreras con menos de un $8 \%$. La licenciatura en sociología agrupa la mayoría de los encuestados en el área de ciencias sociales y humanidades con un $43 \%$, seguido por lingüística con un $26 \%$ e historia con $12 \%$; les siguen otras 5 carreras con un porcentaje menor al $9 \%$ (ver gráfica 1 ).

GRÁFICA 1. Porcentaje de estudiantes por carrera de la UAM-I
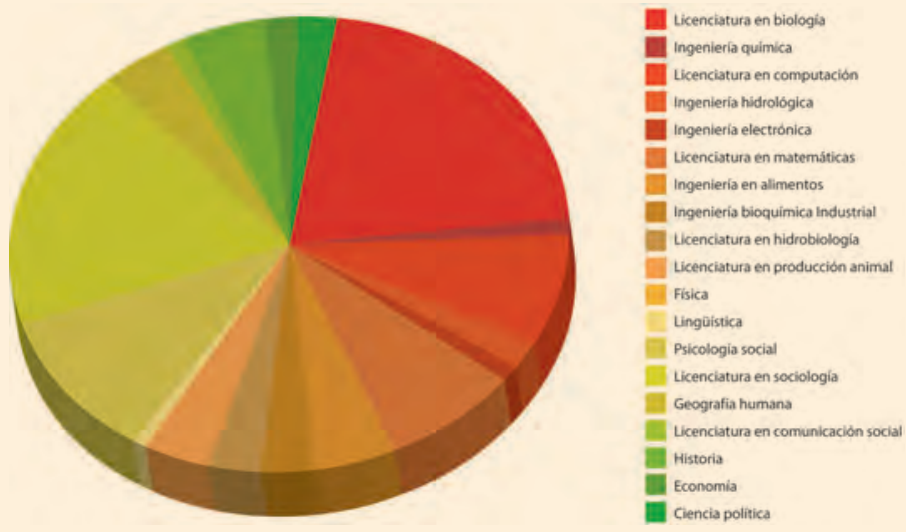

\section{ANÁLISIS DE LOS ESTUDIANTES DE CIENCIAS NATURALES Y EXACTAS DE LA UAM-I}

Casi todos los estudiantes de ciencias naturales y exactas respondieron que sí se consideran personas interesadas en la ciencia (sólo un 5\% dijo lo opuesto). Las publicaciones electrónicas (blogs, redes sociales, páginas de internet, etc.) fue el medio preferido por los estudiantes para informarse de cuestiones científicas, seguido por las revistas y la televisión en tercer lugar. Ante la pregunta de si sabían qué era nanociencia y nanotecnología, más de la mitad de los encuestados (un 54\%) respondió afirmativamente y la mayoría (75\%) dijo tener la idea de que la nanociencia y la nanotecnología tenían que ver con la ciencia y la tecnología aplicada a escala pequeña, aunque debe aclararse que las respuestas en algunas ocasiones eran vagas y poco precisas. Sólo unos pocos mencionaron la escala de $10^{-9}$. Un grupo de encuestados (16\%) dijo tener la idea de que la nanociencia y la nanotecnología era algo vinculado a la fabricación de chips o robots muy pequeños.

Contrasta con lo anterior las respuestas a la pregunta de si conocían, usaban o habían visto algún producto derivado de la nanotecnología, pues $82 \%$ dijo no haber visto alguno de estos productos. Los aparatos electrónicos (como celulares, computadoras, televisiones, reproductores de música, etc.) resultaron ser el grupo de producto más mencionado como ejemplo,seguido por los cosméticos, siendo las tiendas 
especializadas o de alguna marca de productos en específico el lugar donde se ha visto la mayoría de dichos productos.

En lo que refiere al grado de seguridad a la salud y el ambiente percibido, $67 \%$ de los encuestados dijo no estar seguro o no saber, frente a un $28 \%$ que señaló que sí consideraba que son seguros, tal y como muestra la gráfica 2. No sobra precisar que cuando se preguntó el porqué de su respuesta, la mayoría, cuando respondió, aseguró que no sabía. Únicamente el 11\% justificó su respuesta diciendo que no había la información suficiente al respecto o bien, que hacían falta más estudios sobre el tema.

GRÁFICA 2. ¿Por qué los alumnos de las carreras de ciencias naturales y exactas de la UAM-I consideran que los productos nanotecnológicos son o no seguros para la salud y el ambiente.

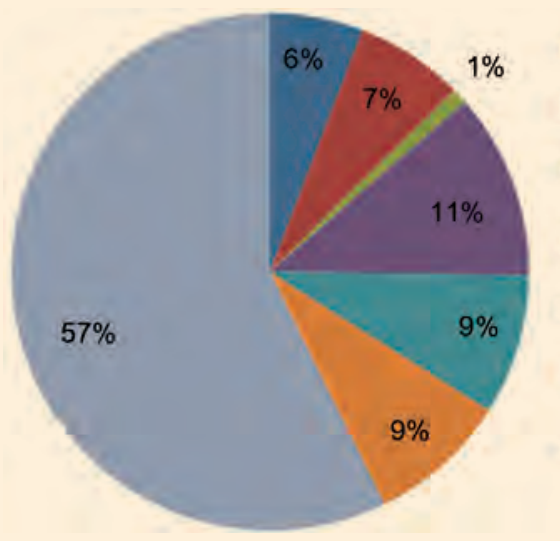

Es bueno para el ambiente, es ecológico

Ayuda a combatir enfermedades

No se han probado del todo sus posibles daños a la salud y/o el ambiente

No hay suficiente información o estudios

Se pueden usar con malos propósitos

Otro

No sabe/No contestó

Respecto a la interrogante sobre si se considera necesario regular el avance de la nanotecnología y la comercialización de sus productos, 69\% respondió que sí. La mayoría (33\%), sin embargo, no supo argumentar por qué era necesaria tal regulación o bien no contestó la pregunta. Los que contestaron sugirieron que los productos nanotecnológicos necesitan regulación por seguridad o por precaución (24\%), que todos los productos necesitan regulación sin importar sus componentes o procedencia $(14 \%)$ o porque el conocimiento científico puede usarse con fines no éticos o para malos propósitos (10\%).

A la pregunta de que si creían que el nivel científico y tecnológico de México es suficiente para poder innovar en nanotecnología, gran número de estudiantes respondió que no era suficiente (59\%), mientras que un 32\% contestó que sí lo era. Al preguntar por qué lo consideraban así, las respuestas estuvieron bastante divididas. Mientras que un 25\% de los encuestados volvió a responder que no sabía, un 22\% justificó su respuesta diciendo categóricamente que la falta de apoyo económico o de inversión en ciencia y tecnología impedía que México innove en nanotecnología. En contraste, $16 \%$ se mostraba relativamente más optimista al señalar que sí existe el talento y los medios necesarios para lograrlo, pero que la falta de apoyo - principalmente económico- es un gran obstáculo y que esto genera una fuga de cerebros. No debe sorprender que esto último esté presente en el consciente colectivo ante la falta de claridad de oportunidades para los jóvenes universitarios de grado y posgrado. 
En cuanto al grado de confianza (gráfica 3), los productos que tuvieron más bajo promedio fueron los cosméticos, los alimentos y las armas, mientras que la producción de energía verde y los textiles recibieron calificación alta. Lo anterior parece explicarse en el hecho de que la potencial situación de exposición humana a un material nanotecnológico, por ingesta o vía tópica, genera desconfianza; en cambio, si ésos no están directamente en contacto con el cuerpo humano y son usados para obtener energía de fuentes renovables, la percepción cambia positivamente. Llama la atención que esto incluso aplique para el avance en área médica.

GRÁFICA 3. Grado de confianza en el uso de productos nano de los alumnos de ciencias naturales y exactas de la UAM-I en una escla del 1 al 10 donde cero es total desconfianza y 10 absoluta confianza.

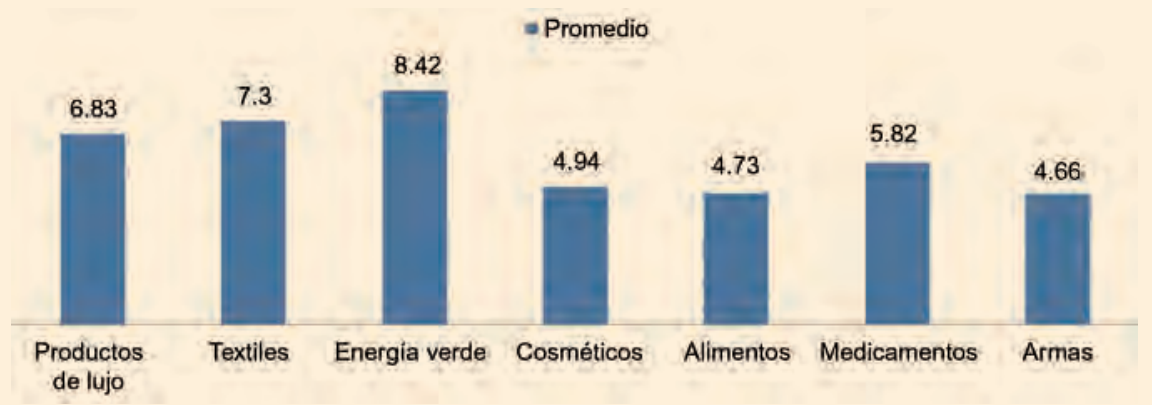

El $81 \%$ de los estudiantes de este grupo dijo estar de acuerdo con etiquetar los productos nanotecnológicos, dando como explicación principal el hecho de que el consumidor tiene derecho a estar informado sobre lo que está consumiendo o comprando. Por último, gran parte de los encuestados se pronunció a favor de regular las importaciones de productos nanotecnológicos. La mayoría justificó su respuesta diciendo que se debe tener un control de lo que entra al país, o bien, refirieron motivos de seguridad.

\section{ANÁLISIS DE LOS ESTUDIANTES DE CIENCIAS SOCIALES Y HUMANIDADES DE LA UAM-I}

El 26\% de este grupo de encuestados manifestó desinterés en la ciencia, aparentemente asumiendo que las sociales y las humanidades "no son ciencias"; 73\% en cambio indicó que sí estaba interesado en las ciencias. Esta diferencia también fue observada entre los alumnos de la UNAM en el estudio de 2011 ya antes mencionado.

En el grupo de sociales y humanidades, muy pocos tuvieron idea de qué es la nanociencia y la nanotecnología: sólo $21 \%$ afirmó saber. Este pequeño grupo, en su mayoría (67\%) la consideró como una tecnología a escala muy pequeña, pero un $17 \%$ señaló que tenía que ver con chips o robots muy pequeños (ciencia ficción), en tanto que $11 \%$ dio una respuesta fuera de contexto o completamente errónea. De igual forma, un $19 \%$ dijo haber visto o usado algún producto derivado de la nanotecnología, 
siendo los aparatos electrónicos el producto más mencionado; la mayoría dijo no saber o no estar seguros del lugar donde vieron a la venta tales productos.

De manera similar al grupo de ciencias naturales y exactas, este segundo grupo se mostró incierto sobre los avances de la nanotecnología para la salud y el ambiente, pues un $78 \%$ respondió que no sabía si eran seguros, al tiempo que 21\% dijo que sí eran seguros y un 1\% sostuvo que no lo eran. En la justificación de las respuestas anteriores, la mayoría optó por no hacerlo o precisar que no sabía. El comportamiento de este grupo fue similar al del grupo anterior. El 54\% de este grupo de ciencias sociales y humanidades se mostró a favor de que se regulen los avances de la nanotecnología y $30 \%$ dijo que no consideraba necesario regular dicho avance. Al preguntar por qué lo consideraban así, poco menos de la mitad no respondió o dijo que no sabía; 26\% señaló que era por seguridad o precaución.

Por otro lado, el 58\% del grupo señaló que México no cuenta con un buen nivel científico y tecnológico frente a un $17 \%$ que respondió que sí (el 25\% restante dijo no saber). Un 32\% señaló que la falta de apoyo económico es lo que impide lo anterior, aunque es de llamar la atención que, de nuevo, un gran número de encuestados (39\%) dijo no saber o simplemente no respondió.

Con respecto al grado de confianza de usar productos nanotecnológicos y usando la misma escala que con el anterior grupo (el "cuadro de confianza"), los productos nanotecnológicos usados en la producción de energía verde es el que mejor promedio con una calificación de 8.22. Los demás productos están en promedios por debajo de 6.7, siendo el desarrollo de armamento, los alimentos y los cosméticos los más bajos (3.68, 5.14 y 5.84 , respectivamente).

Sobre sí los productos nanotecnológicos deben etiquetarse, 69\% contestó que sí, pero de igual forma, $37 \%$ no supo o prefirió no argumentar su respuesta. En cambio $32 \%$ dijo que era para saber bien qué era lo que se estaba consumiendo o comprando.

El 56\% se mostró a favor de regular las importaciones de productos nanotecnológicos y un $26 \%$ señaló que era por medida de seguridad o bien, para tener control de lo que entra al país.

A pesar de la aparente buena disposición de los encuestados de la UAM-I, como podemos ver la gran mayoría optó por dejar varias precisiones sin contestar. Lo dicho en parte respondió al desconocimiento sobre qué es la nanociencia y la nanotecnología y a la dificultad de comunicar y hacer asimilable una explicación informada.

\section{UDLAP}

Los alumnos de la Universidad de las Américas Puebla (UDLAP) conforman un grupo distinto al de la UAM-I pues en su mayoría fueron estudiantes entre los 18 y 22 años: $85 \%$ de la carrera de nanotecnología e ingeniería molecular, 7\% de química, $6 \%$ de bioquímica clínica y $2 \%$ de ciencias farmacéuticas.

Todos los encuestados respondieron considerarse personas interesadas en las ciencias. Las publicaciones electrónicas fueron el principal medio por el cual los estudiantes se informan (76\%), seguidos por las revistas (11\%) y la televisión (7\%). Como ya se mencionó, al ser en gran parte alumnos de la carrera de nanotecnología e ingeniería molecular, la mayoría (93\%)pudo definir de manera clara y precisa qué es la nanociencia y la nanotecnología. Solo $7 \%$ señaló no saber y $4 \%$ dio una respuesta completamente errónea. Esto también se ve reflejado en el hecho de que $76 \%$ con- 
testó que sí han visto productos nanotecnológicos, siendo los cosméticos el grupo de productos más repetido, seguido de los aparatos electrónicos y los productos de limpieza. Las tiendas de autoservicio con un $28 \%$ fueron el lugar donde más se han visto estos productos.

Las respuestas relativas a la seguridad de los avances de la nanotecnología están divididas: mientras $46 \%$ afirma que son seguros, $47 \%$ dice no saberlo y sólo un $7 \%$ dice que no lo son. Resulta interesante cómo los propios alumnos de esta carrera, aunque tienen opiniones divididas, al pedirles justificar su respuesta, la mayoría (30\%)indica que todavía no se han probado del todo los posibles daños a la salud y el ambiente, un $24 \%$ señala que no se ha brindado la información necesaria sobre estos productos y un $15 \%$ afirma que son seguros ya que han sido sometidos a un alto control de calidad.

Sobre la pregunta de si se considera necesario regular el avance de la nanotecnología, 94\% de los encuestados respondió que sí, siendo el principal motivo el hecho de que aún no se sabe el daño que puedan provocar. Más aún, un grupo pequeño dijo que todos los productos, sin importar su contenido, necesitan regulación.

Otra pregunta que dividió opiniones fue la que se refiere a si creen que el nivel científico y tecnológico de México es suficiente para poder innovar en este campo: $50 \%$ dijo que no, pero un $48 \%$ dijo que sí y solo un $2 \%$ dijo no saber. Al preguntarse la justificación de sus respuestas, 39\% dijo que sí hay talento y los medios necesarios pero lo que hace falta es el apoyo económico.

En este grupo de estudiantes de nuevo la producción de energía verde cuenta con un promedio alto en el nivel de confianza de productos nanotecnológicos al registrar una calificación de 9.35. En general, los promedios de todos los productos son altos en comparación con las encuestas aplicadas en otras universidades, aunque de igual forma, los cosméticos, los alimentos y las armas son los que más bajo promedio presentan (6.6, 5.8 y 7.17 respectivamente).

Una gran mayoría (91\%) dijo estar de acuerdo con que se etiqueten los productos nanotecnológicos, siendo los argumentos el que el consumidor tiene derecho a saber lo que está adquiriendo (42\%) y también a saber bien qué tipos de componentes tiene el producto (31\%). El 83\% afirmó que es necesario regular las importaciones de productos nanotecnológicos, esto con el fin de tener un mejor control de lo que entra al país o bien como medida de seguridad (53\%). Sólo 16\% dijo que las importaciones deberían regularse para fomentar la producción y el consumo de productos nacionales.

De manera general, los alumnos de la UDLAP contestaron de modo más informado el cuestionario, motivados tal vez por el hecho de hablar de un tema que conocen. A pesar de ello, es notoria la división existente entre quienes consideran las aplicaciones nanotecnológicas seguras, frente a quienes dicen no saberlo. Pese a todo, es generalizada la idea de que hacen falta más estudios para verificar, en su caso, la posible toxicidad de los productos, así como para informar al público en general.

\section{CINVESTAV NORTE}

En esta sede las respuestas fueron predominantemente femeninas, con un 71\%, contra un $29 \%$ por parte de los hombres. El 100\% de los participantes dijo estar interesado en la ciencia e informarse en su mayoría a través de medios impresos referentes 
a cuestiones científicas. El 75\% mencionó saber lo que es la nanotecnología y nanociencia, obteniendo $60 \%$ de respuestas acertadas o prácticamente perfectas (gráfica 4), ello de acuerdo con las definiciones de referencia utilizadas en la encuesta de 2011 y que refieren a aquellasoficiales propuestas porla Royal Society (Reino Unido), la National Nanotechnology Initiative (EUA) y la de la Oficina Europea de Patentes.

GRÁFICA 4. Respuestas de los alumnos del CINVESTAV Norte sobre qué es la nanociencia y la nanotecnología.

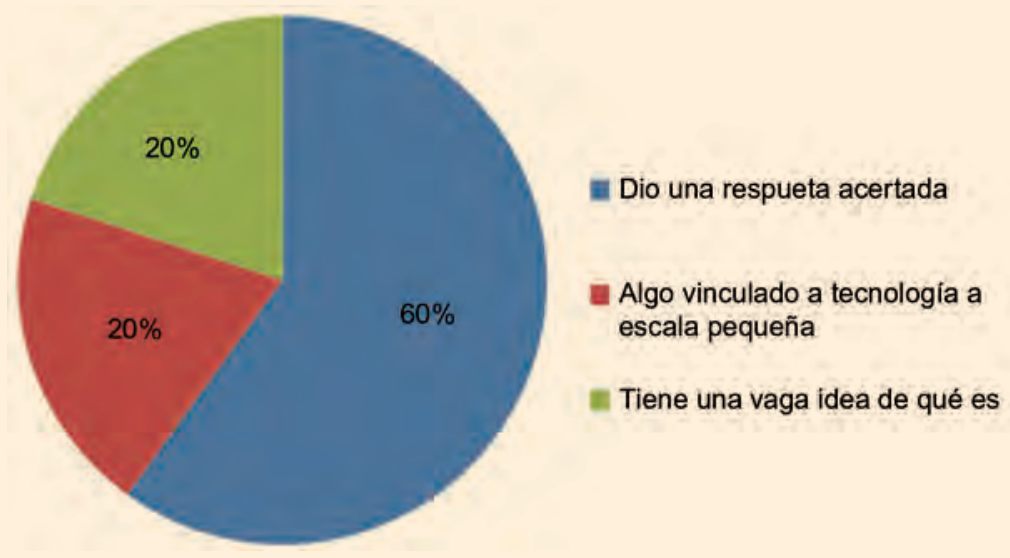

Un $62 \%$ dijo conocer productos nano, añadiendo que éstos consistían en chips, dispositivos médicos y nanomateriales. El área de estudio de los encuestados influyó en la respuesta sobre el lugar donde han visto los productos nano, ya que el $60 \%$ indicó ubicarlos en tiendas especializadas para laboratorios. El 62\% de los encuestados opina que los nanoproductos son seguros para la salud, indicando como motivación para esta idea el que tales productos se encuentran bajo normas de salud y ambiente.

En cuánto a la regulación del uso y comercialización, el 87\% se encuentra a favor, arguyendo que todos los productos, sin importar su origen, deben ser regulados de esta manera, ya sea para evitar accidentes como para generar mayor avance en este rubro de la ciencia y la tecnología. A diferencia de algunas opiniones, que son tajantes al decir que nuestro país no puede innovar en nanotecnología y nanociencia debido a la falta de apoyo económico por parte del Estado, los participantes del CINVESTAV Norte, dicen que existe el potencial científico (gráfica 5), pero que éste se encuentra limitado por los recursos que se le otorga al ámbito de investigación y desarrollo.

La confianza reflejada es mayor en los casos de textiles, energía verde y medicamentos, contra los cosméticos, los alimentos y las armas, que presentan un menor promedio de confianza, tal y como podremos observar en el apéndice que se incluye al final sobre promedio de productos con menor o mayor grado de confianza por parte de los encuestados. Por otra parte, el 75\% opina que los nanoproductos deben ser etiquetados, ello al igual que cualquier otro producto. Para concluir, $62 \%$ opina que la regulación legal es necesaria, entre otras cuestiones, por motivos de seguridad. 
GRÁFICA 5. ¿Crees que el nivel científico y tecnológico de México es suficiente para poder innovar en nanotecnología? Respuestas de los alumnos del CINVESTAV Norte.

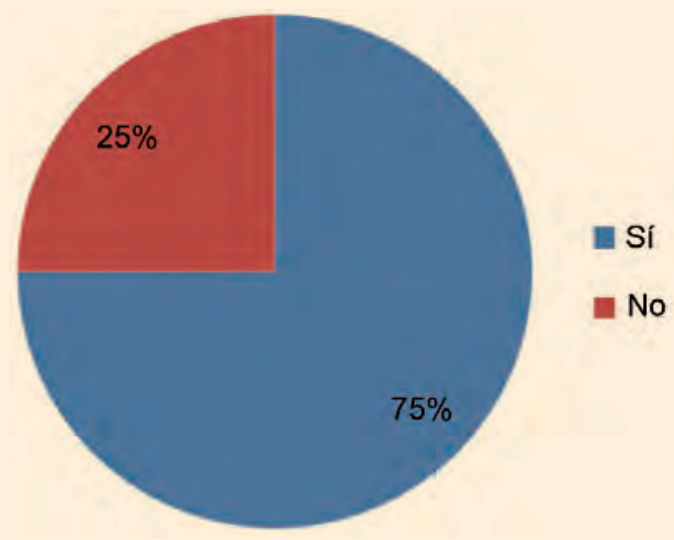

\section{CINVESTAV SUR}

En esta sede hubo más investigadores que colaboraron con nuestro cuestionario, a pesar de ser una de las sedes con menos participantes. Es por eso que el promedio de edad es de 33 años y las carreras están relacionadas con la educación y la pedagogía, existiendo sólo un antropólogo social.

El 79\% se considera interesado en la ciencia y los medios informativos preferidos son las publicaciones electrónicas y la televisión. ${ }^{2}$ El conocimiento y desconocimiento sobre qué es nanociencia y nanotecnología es similar (50-50). De modo similar, 43\%

GRÁFICA 6. Porcentajes de alumnos encuestados por carrera del CINVESTAV Sur.

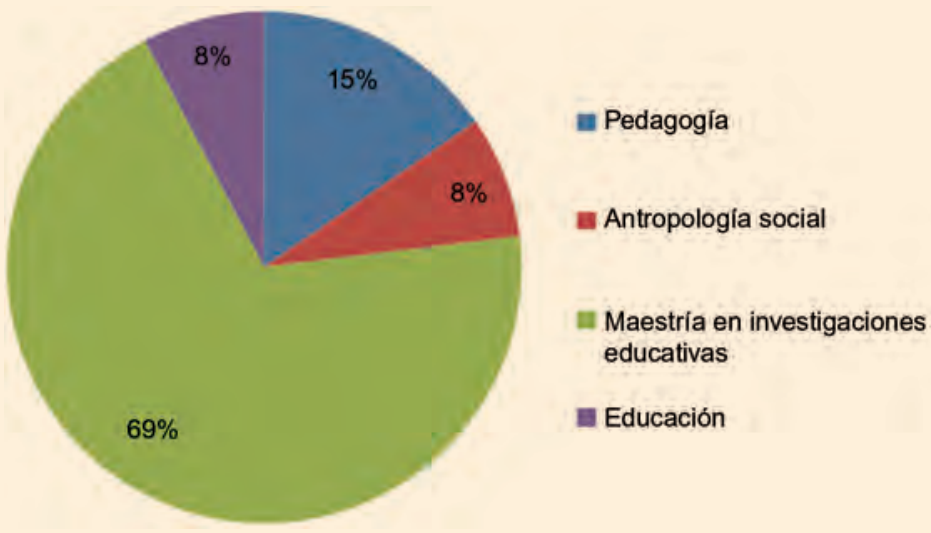

2 Para consultar los porcentajes exactos, ver el apéndice final de cuadros comparativos. 
opina que estos conceptos se refieren a una tecnología a pequeña escala, mientras que $7 \%$ tiene una idea apropiada de lo que es lo nano pero sin ser muy específicos.

El conocimiento y desconocimiento sobre productos nano es igualmente dividido en dos ya que $57 \%$ sostiene haberlos visto, mientras que el $43 \%$ no. Los encuestados que dijeron conocer o haber visto algún "producto nano" (que hacen uso de la nanotecnología) en establecimientos comerciales de diversa índole, refieren que son, en su mayoría aparatos electrónicos y que los han visto en tiendas de electrónica. Respecto a la cuestión de seguridad de los productos, el 57\% no sabe si son seguros y en esa misma proporción no justifican su respuesta o indican no saberlo. También los encuestados argumentan que todos los productos deben ser regulados respecto a su uso y comercialización, con un $72 \%$ y 55\% respectivamente. En este plantel, el 55\% piensa que en nuestro país existe un gran potencial humano, pero que no cuenta con apoyo económico gubernamental apropiado.

El 79\% considera que el etiquetar los productos es importante para estar informados de lo que se consume. El mismo porcentaje opina que también es importante la regulación legal de los productos nano, tanto por ser como los demás productos, como por motivos de seguridad y control que puedan ser específicos a dicho frente tecnológico.

\section{ESCA TEPEPAN}

En este plantel se aplicaron 141 encuestas a una población de entre 18 y 25 años, siendo $66 \%$ mujeres y $34 \%$ hombres. La formación de los encuestados es: contabilidad pública (77\%), relaciones comerciales (22\%) y negocios internacionales (1\%).

Contrario a otras escuelas del Instituto Politécnico Nacional, el 33\% de los individuos manifestó no estar interesado en las ciencias. Los medios de información preferidos fueron las revistas, las publicaciones electrónicas y la televisión. Por otro lado, sólo el 39\% dice que sabe qué es la nanociencia y la nanotecnología: 31\% piensa que es tecnología a escala muy pequeña, $1 \%$ tiene una idea de lo nano y un $7 \%$ dio una respuesta perfecta ante lo que significa nanociencia y nanotecnología. Del 30\% que dijo conocer algún producto nano, el 65\% dijo que éstos eran aparatos electrónicos, el $16 \%$ que eran chips, el 10\% habló de medicamentos y el $5 \%$ de robots. Las respuestas sobre dónde se han visto estos productos fueron muy variadas a diferencia de otras sedes, mencionando farmacias, bancos, escuelas y hospitales, además de la respuesta más recurrente de tiendas de electrónica.

Más adelante, en la pregunta sobre la percepción sobre la seguridad de los productos, el $70 \%$ indicó no saber si los productos nano son o no seguros, $26 \%$ afirmó que sí son seguros y sólo 4\% negó que lo fueran. Las razones de tales respuestas incluyen argumentos como "son [materiales] radioactivos", "son buenos para el ambiente" (6\%) y“depende de las aplicaciones" (8\%), son seguros debido a que si provienen de un estudio científico "deben" ser buenos (8\%).

Denota que $67 \%$ de los encuestados dice que es necesario regular el avance de la nanotecnología y la nanociencia debido, a quecon ello "se genera avance en la ciencia" (18\%), porque debe existir un "control de uso y calidad" (18\%) y ya que hay que "hacerlos más accesibles" a todo el espectro de potenciales consumidores (14\%). El 32\% no contestó o no supo dar una razón a cerca de una eventual regulación de este avance científicotecnológico. 
GRÁFICA 7. Los productos nanotecnológicos, ipor qué considera que son o no son seguros? Respuestas de estudiantes de la ESCA Tepepan.

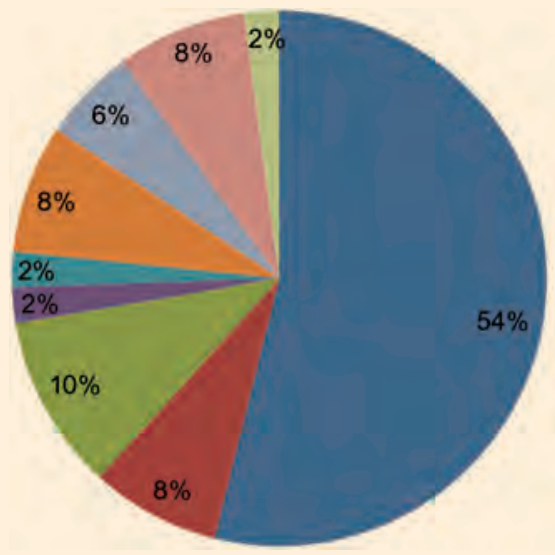

\author{
- No sabe/No contestó \\ - Tienen pros y contras \\ No conozco sobre el tema \\ Son radioactivos \\ - Contaminan muchoi \\ III Depende de sus aplicaciones \\ Son buenos para el ambiente \\ Ir Es ciencia, debe ser bueno \\ Ayudan a cuestiones médicas
}

En lo que respecta a la pregunta referente a si México está en condiciones para innovar en nanociencia y nanotecnología, el 74\% contestó que no, de la misma forma que en los grupos anteriores de encuestados, debido a la falta de inversiones en investigación y desarrollo por parte del Estado.

En el apartado sobre la confianza en los productos nano, el mayor promedio lo tienen la energía verde, los productos de lujo y los textiles, y el menor, las armas, los medicamentos y los alimentos(gráfica 8).

Sobre la necesidad de etiquetar los productos nano, el 70\% de respuestas son afirmativasa pesar de que el 34\% no justificó su posicionamiento. El 16\% habló de la importancia de identificarlos y clasificarlos y un $25 \%$ de etiquetarlos como una manera de conocerlos mejor y familiarizarse con ellos. Finalmente, en lo que respecta a la regulación legal, el 63\% concordó en la necesidad de hacerlo, sobre todo para apoyar a

GRÁFICA 8. Grado de confianza en el uso de productos nano de los alumnos de la ESCA Tepepan en una escala del 1 al 10 donde cero es total desconfianza y 10 es absoluta confianza.

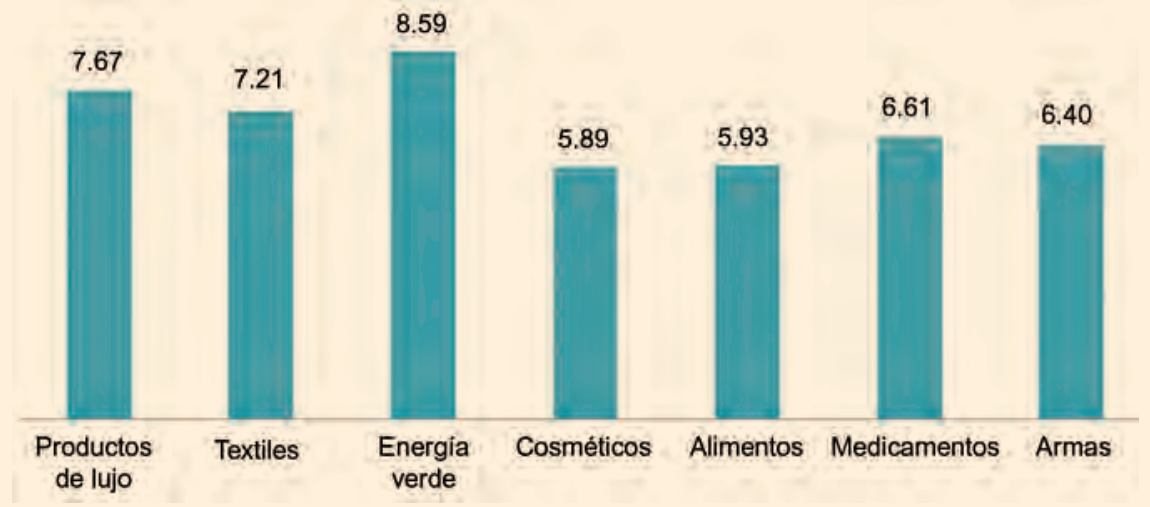


la economía e industrias mexicanas (16\%), controlar la calidad y el uso que se le da a los (nano)productos (11\%) y para promover la nanotecnología (8\%); no obstante, debe señalarse que en este punto $43 \%$ de los encuestados no aclaró sus razones para apoyar o rechazar la regulación de la nanotecnología.

\section{ESIME CulHuACÁN}

En esta entidad se encuestaron a 71 jóvenes entre los 18 y los 25 años de edad, quienes cursan las carreras de ingeniería mecánica e ingeniería en comunicaciones y electrónica siendo $82 \%$ hombres y sólo $18 \%$ mujeres.

Absolutamente todos los sujetos declararon considerarse interesados en la ciencia e informarse por medios electrónicos, publicaciones del IPN y revistas, según el orden de priorización. Un 79\% afirmó saber qué es la nanociencia y la nanotecnología. Se registra también una incidencia mayor en los casos que tienen una idea de lo nano, ya que $26 \%$ mencionaron la escala nanométrica como referencia, mientras que el $47 \%$ presentó la definición que consistía en tecnología a escala pequeña.Sólo 4\% nos brindó una respuesta perfecta, misma cifra que las respuestas fuera de contexto. El 20\% no supo o no contestó el apartado de esa pregunta.

La respuesta negativa, respecto a conocer productos nanotecnológicos fue predominante, con un 61\%, y el 39\% restante que respondió afirmativamente, mencionó desde los aparatos electrónicos, ropa, materiales y aparatos auditivos, hasta los reactores nucleares. Contestaron haberlos visto en tiendas de electrónica y departamentales y, un 5\% afirmó que la nanotecnología se encuentra en todas partes.

En torno a la seguridad de los productos nano, $62 \%$ mencionó que considera que sí lo son, teniendo una amplia variedad de respuestas que presentamos en la gráfica siguiente.

A pesar del ausentismo de respuestas concretas, en el orden del 36\% del total, los encuestados que respondieron brindaron una amplia visión de sus perspectivas que,

GRÁFICA 9. Los productos nanotecnológicos, ¿por qué considera que son o no son seguros? Respuestas de los alumnos de la ESIME Culhuacán.

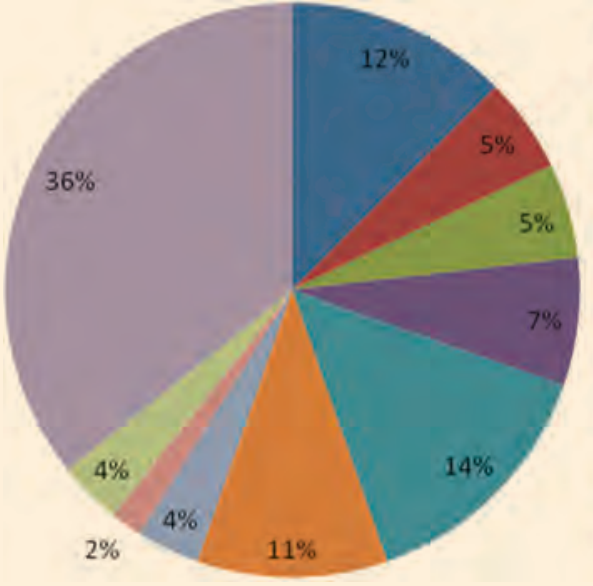

Es ciencia, debe ser bueno

Reducen costos y dan beneficios

Ayudan a las cuestiones médicas

Por su tamaño tan pequeño no afectan

Tienen pros y contras

Depende de las aplicaciones

Son buenos para el ambiente

Están probados

Salvan vidas

No sabe/No contestó 
según nos muestran, se encuentran más favorables y muestran más confianza en esta tecnologíao al menos más abiertos al conocimiento de la misma que las opiniones halladas en otras sedes y que manifestaban opiniones cautelosas. Ejemplo de ello se observa en la gráfica 9, donde el $14 \%$ dice que éstos productos tienen tanto pros como contras, el $12 \%$ dice que si son producto de la ciencia deben ser beneficos, el 11\% habla de que la confianza dependerá de las aplicaciones de tales productos, un 7\% manifiesta que el tamaño tan pequeño de los componentes los hace inofensivos;otro $5 \%$ sostiene que se reducen costos y aportan beneficios al tiempo que otro $5 \%$ alude a que ayudan en cuestiones médicas. Un $4 \%$ dice que tienen la característica de salvar vidas y un $2 \%$ alega que están probados. Sólo un $4 \%$ evitó responder la pregunta.

En este plantel también se percibe que México no tiene un nivel suficiente como para innovar en nanotecnología (72\%) debido a que, no hay apoyo económico suficiente por parte del gobierno (40\%), porque el potencial existente es limitado (14\%) y debido a la falta de interés en la ciencia (18\%).

Como en todos los casos anteriores,las aplicaciones en energía verde se consideran merecedoras de mayor confianza con un promedio de 8.6, seguidas por las de los textiles con 7.14 y los productos de lujo con 6.88. En cambio, las aplicaciones en armas (6.04) y alimentos (5.23) tienen el menor promedio de confianza, ello superados por los cosméticos con un promedio de 4.8.

Respecto al etiquetado de productos un 79\% está de acuerdo en que es necesario, de acuerdo con lo que muestra la gráfica 10.

GRÁFICA 10. ¿Por qué los alumnos de la ESIME Culhuacán consideran que se debe o no regular los productos nanotecnológicos?

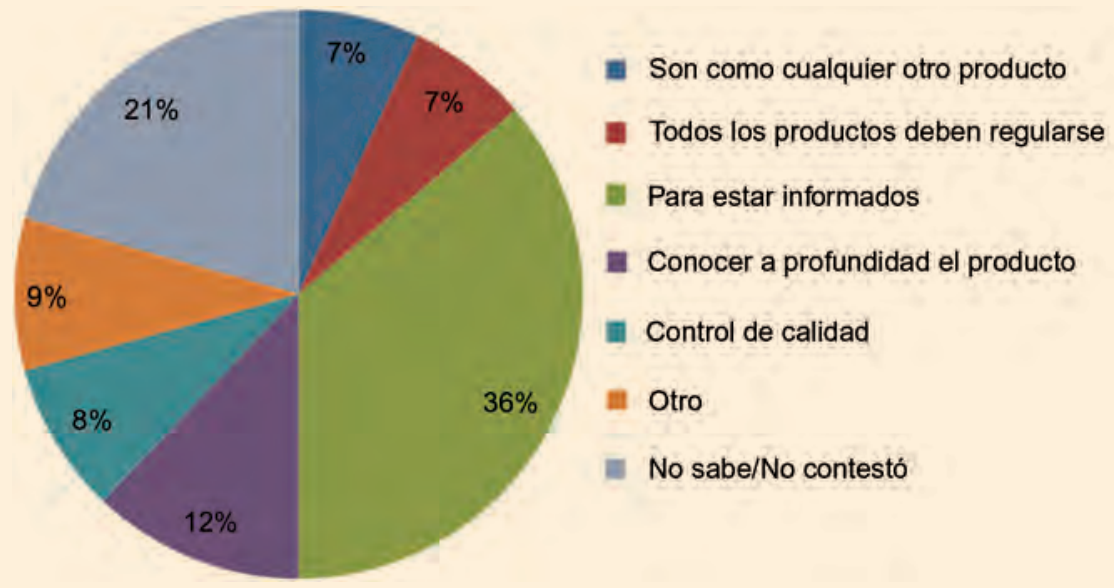

El 79\% de los participantes están a favor de la regulación legal de las importaciones respecto a los productos nanotecnológicos por que según se percibe, así se tendría un mayor control sobre el uso y distribución (22\%) al tiempo que se apoyaría el mercado y la industria nacional (15\%). 


\section{RefLeXIONeS finales}

El contacto directo con los estudiantes y docentes universitarios y la percepción que tienen sobre la nanociencia y la nanotecnología corrobora que si bien es cierto que hay un número amplio de personas que conocen este frente científicotecnológico, aún quedan muchos sectores en la esfera del desconocimiento o la falta de información adecuada. Sin embargo, denota que pese al acceso a la información e independientemente de la precisión de la misma, los resultados de percepción son muy parecidos en lo que respecta a la confianza y necesidad de regualción de los nanoproductos. En este sentido, los resultados coiciden en términos generales con las tendencias expuestas en el metanalisis de estudios de percepción social realizado por Satterfield et al. (2009) para el caso de países desarrollados.

Ahora bien, debe notarse que se trata de un universo de encuestados que supone ser el más leído y formado, por lo que se puede sostener que el conocimiento social sobre la temática es mayoritariamente reducido en nuestro país. La importancia de este trabajo está entonces en dar cuenta de ello, todo al tiempo que se indagan las percepciones sociales existentes - correctas e incorrectas - para poder así contar con un análisis del contexto social en y desde el cual se innova. Lo dicho ayuda a develar la importancia de lo social, no sólo en términos de aceptación o rechazo de las nuevas tecnologías, sino también en los procesos de asimilación y diseminación social de las mismas y, más áun, en la construcción sociopolítica de la agenda científica y tecnológica en tanto fundamento de la reducción práctica de costos innecesarios, tanto económicos como sociales. Sostenemos por tanto que la necesidad de estimular la comunicación, divulgación, diálogo participativo y concientización sobre la nanociencia y las nanotecnologías, sus retos, avances, implicaciones.

Consideramos el presente como un trabajo no conclusivo, abierto a la profundización de la búsqueda y análisis de informaciónmás extensa y cada vez más fina. No obstante, esperamos contribuir con esta entrega a la génesis de nuevos estudios sobre la sociedad y su percepción de las ciencias y las nuevas tecnologías, tanto en otros estados de nuestro país como a nivel nacional y regional. Tales estudios bien podrían contribuir en la promoción de un fructífero diálogo y alianzas que procuren fomentar, tanto el avance responsable de la ciencia y las tecnologías, como la participación social en asuntos científicotecnológicos que a corto, mediano o largo plazo, formarán, quizá, parte de nuestra vida cotidiana. Ello, sin embargo, no será lo suficientemente relevante si en el país no se destinan recursos mínimos a la educación, a la ciencia y a la innovación tecnológica.

\section{Agradecimientos}

Agradecemos y damos crédito al proyecto LAB-nano (CEIICH-CONACyT).También expresamos nuestro agradecimiento a Alejandra Azucena Pozos Tovar, de laFacultad de Química de la UNAM por su colaboración y ayuda en el vaciado de encuestas y realización de gráficas, así como a Jacqueline Calderón Hinojosa, estudiante de la Facultad de Filosofía y Letras de la UNAM por su colaboración y ayuda tanto en la aplicación como por su colaboración y observaciones. 


\section{APÉNDICE}

GRÁFICA 11. Porcentajes sobre regulación de uso de nanomateriales y su comercialización en la UAM-I.

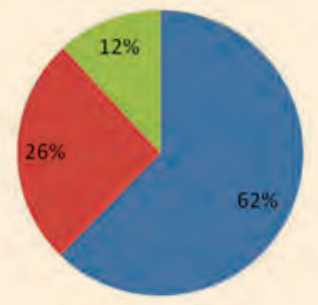

$$
\begin{aligned}
& \text { Sidebe } \\
& \text { regularse } \\
& \text { No debe } \\
& \text { regularse } \\
& =\text { Nosabe/No } \\
& \text { contestó }
\end{aligned}
$$

GRÁFICA 13. Porcentajes sobre regulación de uso de nanomateriales y su comercialización en la UDLAP.

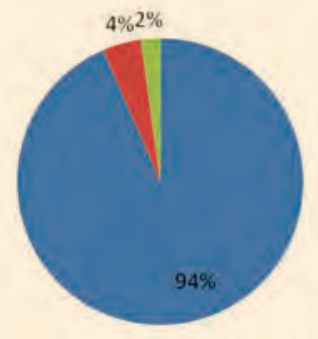

$$
\begin{aligned}
& \text { - Sidebe } \\
& \text { regularse }
\end{aligned}
$$$$
\text { no debe }
$$$$
\text { regularse }
$$$$
\text { a No sabe/No }
$$$$
\text { contestó }
$$

GRÁFICA 15. Porcentajes sobre regulación de uso de nanomateriales y su comercialización en sedes del IPN.

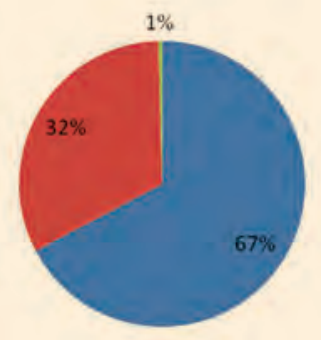

Sidebe regularse

No debe regularse

no sabe/No contestó
GRÁFICA 12. Porcentajes sobre regulación de importaciones de productos nano en la UAM-I.

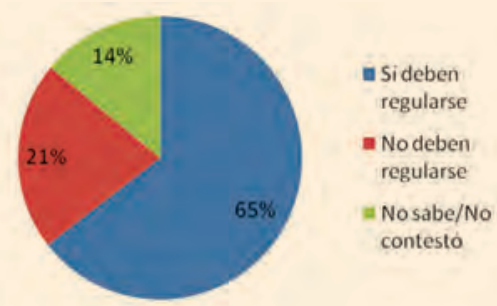

GRÁFICA 14. Porcentajes sobre regulación de importaciones de productos nano en la UDLAP.

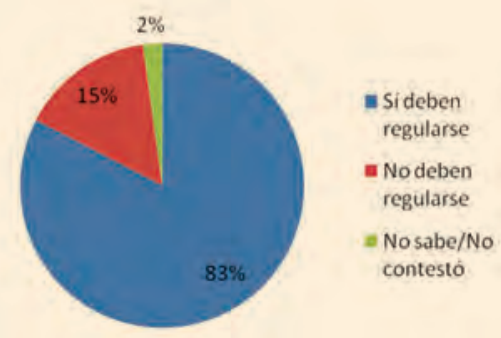

GRÁFICA 16. Porcentajes sobre la opinión acerca de la regulación de importaciones en productos nano en sedes del IPN.

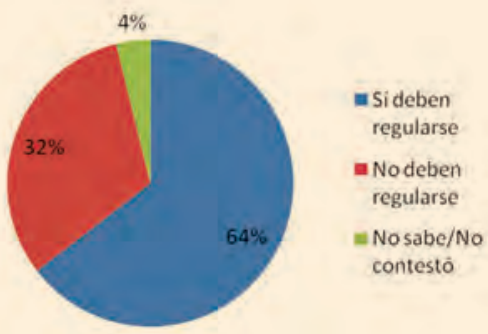


GRÁFICA 17. Grado de confianza en el uso de productos nano del total de alumnos escuestados de la UAM-I en una escala del 1 al 10 donde cero es total desconfianza y 10 es absoluta confianza.

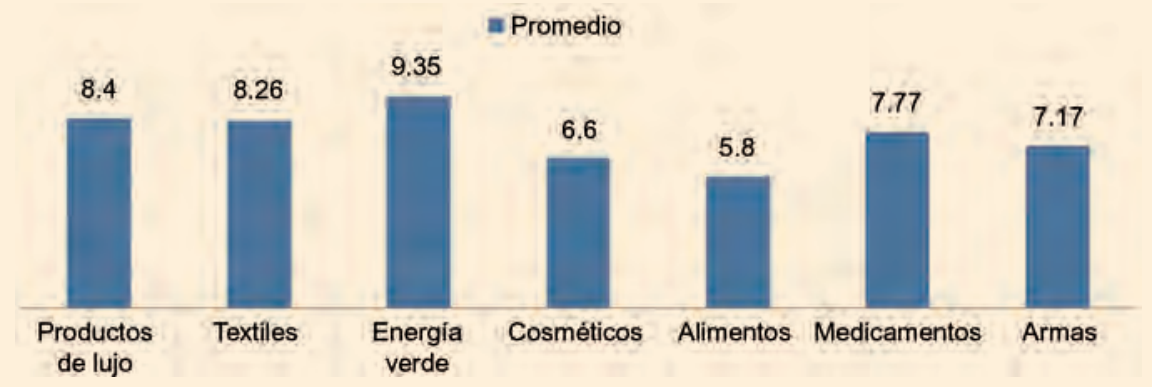

GRÁFICA 18. Grado de confianza de productos nano del total de alumnos escuestados del IPN en una escala del 1 al 10 donde cero es total desconfianza y 10 es absoluta confianza.

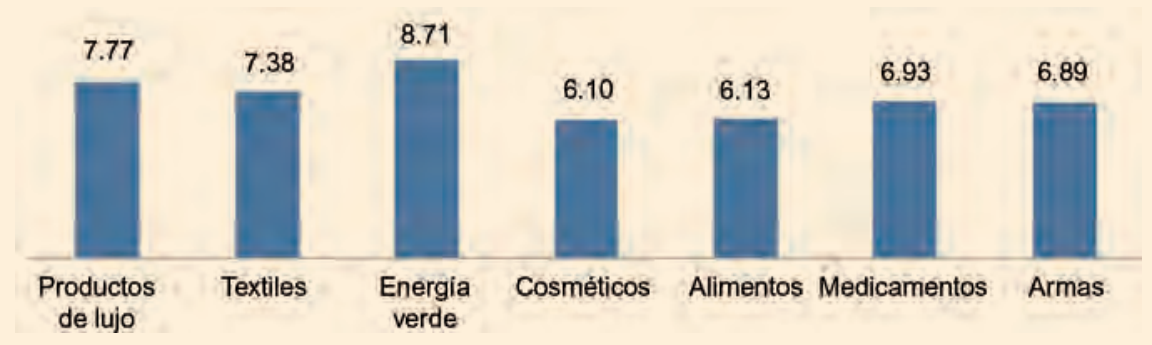

\section{Referencias:}

Delgado Ramos, Gian Carlo y Peña Jiménez, Judith Sarai, (2011). “Análisis de percepción sobre la nanociencia y la nanotecnología: el caso de la comunidad universitaria de la UNAM." Mundo Nano. Revista Interdisciplinaria en Nanociencia y Nanotecnologí,vol. 4,núm. 1, enero-junio, México: 8597.

Satterfield, et al. (2009). "Anticipating the perceived risk of nanotechnologies". NatureNanotechnology, vol. 4, noviembre: 752 - 758. 